\title{
Respostas agronômicas da pimenta malagueta a doses de nitrogênio
}

\author{
Agronomic responses of chili peppers to doses of nitrogen
}

\section{Ricardo Barros Silva ${ }^{1 \star}$, Wemerson Saulo da Silva Barbosa ${ }^{1}$, Wellington Manoel dos Santos ${ }^{1}$, João Cardoso de Albuquerque Neto ${ }^{1}$, Antônio Lucrécio dos Santos Neto ${ }^{1}$, Ademária Aparecida de Souza ${ }^{1}$, Gleica Maria Correia Martins ${ }^{2}$, José Dailson Silva de Oliveira ${ }^{1}$}

\author{
${ }^{1}$ Universidade Federal de Alagoas, Arapiraca, AL, Brasil. Autor para correspondência: ricardoufal2010@gmail.com \\ ${ }^{2}$ Instituto Federal de Educação, Ciência e Tecnologia de Alagoas, Penedo, AL, Brasil.
}

Submissão: 13/01/2020 | Aceite: 14/12/2020

\begin{abstract}
RESUMO
A pimenta malagueta possui grande importância econômica e social para produtores devido sua crescente procura por agroindústrias que confeccionam molhos. Apesar disso, estudos sobre o manejo nutricional da pimenta malagueta são escassos, logo a adubação em cultivos é realizada, principalmente, de forma empírica. Diante disso, este trabalho objetivou verificar a influência de doses de nitrogênio na produtividade de pimenta malagueta. O experimento foi conduzido entre agosto de 2016 e maio de 2017 na área experimental do Campus Arapiraca, da Universidade Federal de Alagoas, Arapiraca, AL. O delineamento experimental utilizado foi em blocos casualizados, com quatro repetições, e seis doses de nitrogênio $\left(0,100,200,300,400\right.$ e $\left.500 \mathrm{~kg} \mathrm{ha}^{-1} \mathrm{~N}\right)$. A aplicação do $\mathrm{N}$ ocorreu de forma parcelada aos 108 , 142,172 e 202 dias após o plantio (DAP), utilizou-se ureia como fonte de N. A colheita de frutos iniciou-se 90 DAP, sendo realizada em uma frequência semanal. As variáveis avaliadas foram: altura de planta $(\mathrm{m})$, diâmetro do dossel $(\mathrm{cm})$, índice SPAD, número de frutos e produtividade $\left(\mathrm{t} \mathrm{ha}^{-1}\right)$. As doses não influenciaram na altura de plantas e diâmetro do dossel. O maior índice $\operatorname{SPAD}(53,11)$, maior produção de frutos totais ao final do período de colheita (2.773 frutos por planta $\left.{ }^{-1}\right)$ e maior produtividade $\left(26,25 \mathrm{t} \mathrm{ha}^{-1}\right)$ foram verificados com $500 \mathrm{~kg} \mathrm{ha}^{-1}$ de $\mathrm{N}$.
\end{abstract}

PALAVRAS-CHAVE: Capsicum frutescens, manejo nutricional, adubação nitrogenada, produtividade.

\begin{abstract}
Chili peppers hold great economic and social importance for producers due to growing demand from agribusinesses that make sauces with them. Nevertheless, studies on the nutritional management of chili peppers are scarce. Thus, fertilization of the crop is mainly empirical. This study was conducted to verify the influence that doses of nitrogen have on the yield of chili peppers. The experiment was conducted between August 2016 and May 2017 in the experimental area of the Arapiraca Campus of the Federal University of Alagoas in Arapiraca, Alagoas. A randomized block design with four replications was employed for six doses of nitrogen $\left(0,100,200,300,400\right.$, and $\left.500 \mathrm{~kg} \mathrm{ha}^{-1} \mathrm{~N}\right)$. Nitrogen was applied in increments 108, 142, 172, and 202 days after planting (DAP); urea was the source of N. Harvesting began 90 DAP and on a weekly basis. The following variables were observed: plant height $(\mathrm{m})$, canopy diameter $(\mathrm{cm})$, SPAD index, number of fruits, and yield $\left(\mathrm{t} \mathrm{ha} \mathrm{H}^{-1}\right)$. The doses did not affect plant height and canopy diameter. The highest SPAD index (53.11), the greatest total fruit production by the end of the harvest period $\left(2,773\right.$ fruits per plant $\left.{ }^{-1}\right)$, and the greatest yield $\left(26.25 \mathrm{t} \mathrm{ha}^{-1}\right)$ were verified with $500 \mathrm{~kg} \mathrm{ha}^{-1}$ of N.
\end{abstract}

KEYWORDS: Capsicum frutescens, nutrition management, nitrogen fertilization, yield.

\section{INTRODUÇÃO}

A pimenteira é uma planta pertence à família Solanaceae, que é cultivada em todo território nacional. De acordo com a Organização das Nações Unidas para Alimentação e Agricultura - FAO (2017), na safra de 2017, a produção mundial de pimenta correspondeu a 6,90 milhões de toneladas, numa área colhida de 568 mil hectares, com produtividade média de $12,15 \mathrm{t} \mathrm{ha}^{-1}$. Nesse mesmo ano, a área plantada no Brasil foi de 28.631 hectares, com produtividade média de $27,72 \mathrm{t} \mathrm{ha}^{-1}$, o que resultou em uma produção de 793.70 toneladas.

Em Alagoas, o cultivo de pimenta aumentou na última década, principalmente, o cultivo da pimenta 
ardida, conhecido como malagueta. Esse aumento no cultivo ocorreu em função da crescente demanda no Nordeste, principalmente em estados como Pernambuco e Sergipe, onde a demanda agroindustrial desse tipo de matéria-prima vem ganhando destaque para confecção de molhos ardidos.

Apesar de existirem estudos sobre diversos tipos de pimenta em resposta a adubação nitrogenada (EKWU et al. 2002, AYODELE et al. 2015), ainda são poucas as pesquisas voltadas para o tipo malagueta, tanto a nível nacional, quanto a regional, o que dificulta a adoção de manejo adequado da adubação nitrogenada em cultivos, isso interfere na produtividade de frutos e, consequentemente, na rentabilidade da cultura.

O nitrogênio $(\mathrm{N})$ caracteriza-se como um dos elementos exigidos em maior quantidade por espécies da família Solanaceae, porque participa diretamente do crescimento das plantas, floração e frutificação. É elemento estrutural de componentes celulares em vegetais, como: ácidos nucléicos, proteínas e clorofila (TAIZ et al. 2017, MAHMUD et al. 2020). Apesar disso, não há indicação de dose de $\mathrm{N}$ de máxima eficiência agronômica na cultura da pimenta malagueta, em Alagoas. Assim, a adubação é realizada quase sempre de forma inadequada, acarretando excessos e desperdício de fertilizantes nitrogenados. Logo, a obtenção do melhor manejo para a adubação nitrogenada da cultura pode contribuir de forma considerável em cultivos da região Nordeste e do estado de Alagoas.

Diante do exposto, objetivou-se verificar a influência de doses de $\mathrm{N}$ em características agronômicas da pimenta malagueta no agreste alagoano.

\section{MATERIAL E MÉTODOS}

\section{Caracterização da Área Experimental}

O experimento foi conduzido na área experimental do Campus Arapiraca da Universidade Federal de Alagoas, na cidade de Arapiraca (9 45’58" S; 35 38' 58” W; 264 m), Agreste de Alagoas. O período do cultivo foi de 18 agosto de 2016 a 19 de maio de 2017. NIMER (1977) caracteriza o clima da região com temperatura média anual de $25^{\circ} \mathrm{C}$, precipitação pluvial anual média entre 750 e $1000 \mathrm{~mm}$, onde $50 \%$ da chuva anual concentra-se nos meses de maio, junho e julho, sendo a primavera e o verão o período seco da região.

O solo da área experimental foi classificado como um Latossolo Vermelho distrófico (EMBRAPA 2013). Antes da implantação do experimento, realizou-se a coleta de amostras de solo na camada de 0 a 20 $\mathrm{cm}$ para análise química. Na Tabela 1 encontram-se os atributos químicos e físicos do solo da área experimental. O preparo do solo para o plantio foi realizado de forma mecanizada, com aração e gradagem.

Tabela 1. Características químicas e físicas do solo da área experimental.

Table 1. Chemical and physical characteristics of the soil of the experimental area.

\begin{tabular}{|c|c|c|c|c|c|c|c|c|c|c|}
\hline \multicolumn{11}{|c|}{ Atributos Químicos } \\
\hline $\mathrm{pH}$ & $\mathrm{K}^{+}$ & $P$ & $\mathrm{Na}^{+}$ & $\mathrm{Ca}^{2+}$ & $\mathrm{Mg}^{\circ}$ & $\mathrm{Al}^{3+}$ & $\mathrm{H}+\mathrm{Al}$ & CTC & SB & $\overline{\mathrm{V}}$ \\
\hline \multicolumn{4}{|c|}{------ mg dm ${ }^{-3}$------ } & \multicolumn{4}{|c|}{------- $\mathrm{cmol}_{\mathrm{c}} \mathrm{dm}^{-3}$} & \multicolumn{3}{|r|}{$\%$} \\
\hline 5,6 & 109 & 11 & 12 & $?, 1$ & 0,8 & 1,09 & ,2 & 2,62 & 2,53 & 67,8 \\
\hline \multicolumn{11}{|c|}{ Atributos Físicos } \\
\hline Areia & Silte & \multicolumn{2}{|c|}{ Argila } & \multicolumn{4}{|c|}{ Porosidade } & \multicolumn{3}{|c|}{ Densidade Aparente } \\
\hline \multicolumn{4}{|c|}{$\mathrm{g} \mathrm{kg}^{-1}$} & \multicolumn{4}{|c|}{$\%$} & \multicolumn{3}{|c|}{$\mathrm{g} \mathrm{cm}^{-3}$} \\
\hline 825,6 & 70,5 & \multicolumn{2}{|c|}{103,9} & \multicolumn{4}{|c|}{38,10} & \multicolumn{3}{|c|}{1,33} \\
\hline
\end{tabular}

\section{Delineamento Experimental}

Adotou-se o delineamento experimental em blocos casualizados, com quatro repetições. Os tratamentos foram doses de $\mathrm{N}: 0,100,200,300,400$ e $500 \mathrm{~kg} \mathrm{ha}^{-1}$. Utilizou-se ureia como fonte de nitrogênio. As parcelas eram constituídas por quatro linhas de cultivo no espaçamento de 1,0 (entre linhas) $x$ 0,6 (entre plantas), totalizando 16 plantas por parcelas, numa área de 9,6 $\mathrm{m}^{2}$. A área útil correspondeu a porção central das parcelas $\left(2,4 \mathrm{~m}^{2}\right)$. A área experimental foi equivalente a $192 \mathrm{~m}^{2}$.

\section{Aplicação dos Tratamentos e Manejo da Cultura}

As mudas de pimenta malagueta foram produzidas em bandejas de 128 células, sendo transplantadas quando apresentavam de 4 a 6 folhas, e $10 \mathrm{~cm}$ de altura. Na adubação de fundação aplicouse $300 \mathrm{~kg} \mathrm{ha}^{-1} \mathrm{P}_{2} \mathrm{O}_{5}$ e $240 \mathrm{~kg} \mathrm{ha}^{-1}$ de $\mathrm{K}_{2} \mathrm{O}$, utilizando-se como fonte o superfosfato triplo $\left(45 \%\right.$ de $\left.\mathrm{P}_{2} \mathrm{O}_{5}\right)$ e cloreto de potássio $\left(57,8 \%\right.$ de $\left.\mathrm{K}_{2} \mathrm{O}\right)$, respectivamente, de acordo com o resultado da análise química do solo 
e recomendação de PINTO et al. (1999). Os fertilizantes foram distribuídos em sulcos com $10 \mathrm{~cm}$ de profundidade, distanciados a $15 \mathrm{~cm}$ da linha de cultivo.

A adubação de cobertura nitrogenada (tratamentos) foi dividida em quatro aplicações $(108,142,172 \mathrm{e}$ 202 dias após o plantio). Aliada a primeira adubação de cobertura, aplicou-se mais $50 \mathrm{~kg} \mathrm{ha}^{-1}$ de $\mathrm{K}_{2} \mathrm{O}$.

A irrigação do cultivo foi realizada por gotejamento. Foi utilizado gotejadores espaçados a $20 \mathrm{~cm}$, com vazão média de $1,0 \mathrm{~L} \mathrm{~h}^{-1}$. O turno de rega foi diário em função da evapotranspiração da cultura $-\mathrm{ET}_{\mathrm{C}}$ (mm $\mathrm{d}^{-1}$ ) estimada de acordo com o Boletim FAO 56 (Eq. 1) (ALLEN et al. 1998):

$$
E T C=E T o x K C
$$

em que, $\mathrm{ET}_{0}$ é evapotranspiração de referência estimada pelo método de Penman Monteith - FAO $\left(\mathrm{mm} \mathrm{d}^{-1}\right)$; Kc é o coeficiente da cultura de acordo com DOORENBOS \& KASSAM (1986).

O coeficiente da cultura variou de acordo com a fenologia da pimenteira, onde adotou-se: 0,65 (estágio inicial de desenvolvimento), 0,50 (desenvolvimento vegetativo), 1,00 (frutificação) e 0,80 (maturação dos frutos).

O controle de plantas invasoras durante o cultivo foi realizado através de capinas manuais. Houve o ataque de formigas saúvas, sendo controladas com iscas a base de sulfluramida.

\section{Colheita e Variáveis Avaliadas}

A colheita de frutos iniciou-se 90 DAP, com frequência semanal. Avaliaram-se as seguintes variáveis durante o período de colheita: altura de plantas $(\mathrm{m})$, diâmetro do dossel $(\mathrm{cm})$, índice SPAD, número de frutos por planta e produtividade $\left(\mathrm{t} \mathrm{ha}^{-1}\right)$.

Na obtenção da altura de plantas utilizou-se uma trena métrica, medindo-se as plantas da área útil do nível do solo até sua extremidade superior. O diâmetro da copa foi obtido através de medidas com fita métrica. O índice SPAD foi medido 180 DAP, através de clorofilômetro (Chlorophyll Meter Spad - 502 Plus), em cinco folhas do terço superior da planta, obtendo-se um valor médio. Nas avaliações de número de frutos e produtividade, a cada colheita os dados foram obtidos por contagem e pesagem por meio de balança analítica de precisão $(0,001 \mathrm{~g})$.

\section{Análise Estatística}

Os dados obtidos foram submetidos a análise de variância através do software estatístico Sisvar. As variáveis significativas pelo teste $\mathrm{F}$ a $5 \%$ de significância, foram submetidos a análise de regressão.

\section{RESULTADOS E DISCUSSÃO}

$\mathrm{O}$ efeito das doses de $\mathrm{N}$ nas características produtivas da pimenta malagueta encontra-se descrito na Tabela 2. Verificou-se efeito das doses de $\mathrm{N}$ em produtividade, índice SPAD e número de frutos por planta.

Tabela 2. Análise de variância para produtividade (PROD), altura de plantas (AP), índice SPAD, diâmetro do dossel (DIAD) e número de frutos por planta (NF), na cultura da pimenta malagueta em função de doses de nitrogênio.

Table 2. Analysis of variance for yield (PROD), plant height (AP), SPAD index, canopy diameter (DIAD), and number of fruits per plant (NF) of the chili pepper crop as a function of the doses of nitrogen.

\begin{tabular}{ccccccc}
\hline & & & \multicolumn{4}{c}{ QUADRADOS MÉDIOS } \\
\hline FONTE DE VARIAÇÃO & GL & PROD & AP & SPAD & DIAD & NF \\
\hline Doses de Nitrogênio & 5 & $19,593^{*}$ & $0,003^{\text {ns }}$ & $1247,986^{*}$ & $65,736^{\text {ns }}$ & $144500,119^{*}$ \\
Regressão Linear & 1 & $41,479^{*}$ & $0,00009^{\text {ns }}$ & $1143,56^{*}$ & $63,175^{\text {ns }}$ & $40777,501^{*}$ \\
Regressão Quadrática & 1 & $5,518^{\text {ns }}$ & $0,016^{\text {ns }}$ & $33,972^{*}$ & $58,166^{\text {ns }}$ & $7489,885^{\text {ns }}$ \\
Desvio Regressão & 3 & $16,989^{\text {ns }}$ & $0,00003^{\text {ns }}$ & $23,483^{\text {ns }}$ & $69,113^{\text {ns }}$ & $102412,587^{\text {ns }}$ \\
Blocos & 3 & $0,722^{\text {ns }}$ & $0,018^{\text {ns }}$ & $38,525^{\text {ns }}$ & $73,944^{\text {ns }}$ & $30142,412^{\text {ms }}$ \\
Resíduo & 15 & 4,088 & 0,108 & 80,524 & 37,767 & 37793,56 \\
\hline CV $(\%)$ & 23 & 8,21 & 10,20 & 4,96 & 5,44 & 7,53 \\
\hline
\end{tabular}

${ }^{\text {ns }}$ não significativo e *significativo a $5 \%$ de probabilidade pelo teste $F(p<0,05)$.

A altura de plantas e diâmetro da copa da pimenta malagueta não foram significativas. A altura de plantas média geral foi de $88,37 \mathrm{~cm}$ entre as doses de $\mathrm{N}$ estudadas. Enquanto, o diâmetro do dossel médio geral foi equivalente a $91,48 \mathrm{~cm}$. FURLAN et al. (2015) não obtiveram resultado significativo em variáveis de crescimento na pimenta malagueta em resposta a doses de N. Os autores afirmam que maiores doses de $\mathrm{N}$ tendem a atuar sobre componentes de produção na pimenta malagueta, porém, não interferem de forma significativa em seu crescimento, possivelmente por características endógenas da planta. 
A produtividade da pimenteira respondeu com incremento linear às doses de $\mathrm{N}$ (Figura 1). A dose de $500 \mathrm{~kg} \mathrm{ha}^{-1}$ de $\mathrm{N}$, resultou na maior produtividade de $26,25 \mathrm{t} \mathrm{ha}^{-1}$, enquanto a menor produtividade foi observada na menor dose (testemunha), atingindo um rendimento médio de $20,58 \mathrm{t} \mathrm{ha}^{-1}$, correspondendo a um desempenho menor em $21,60 \%$ quando comparado a maior produtividade obtida.

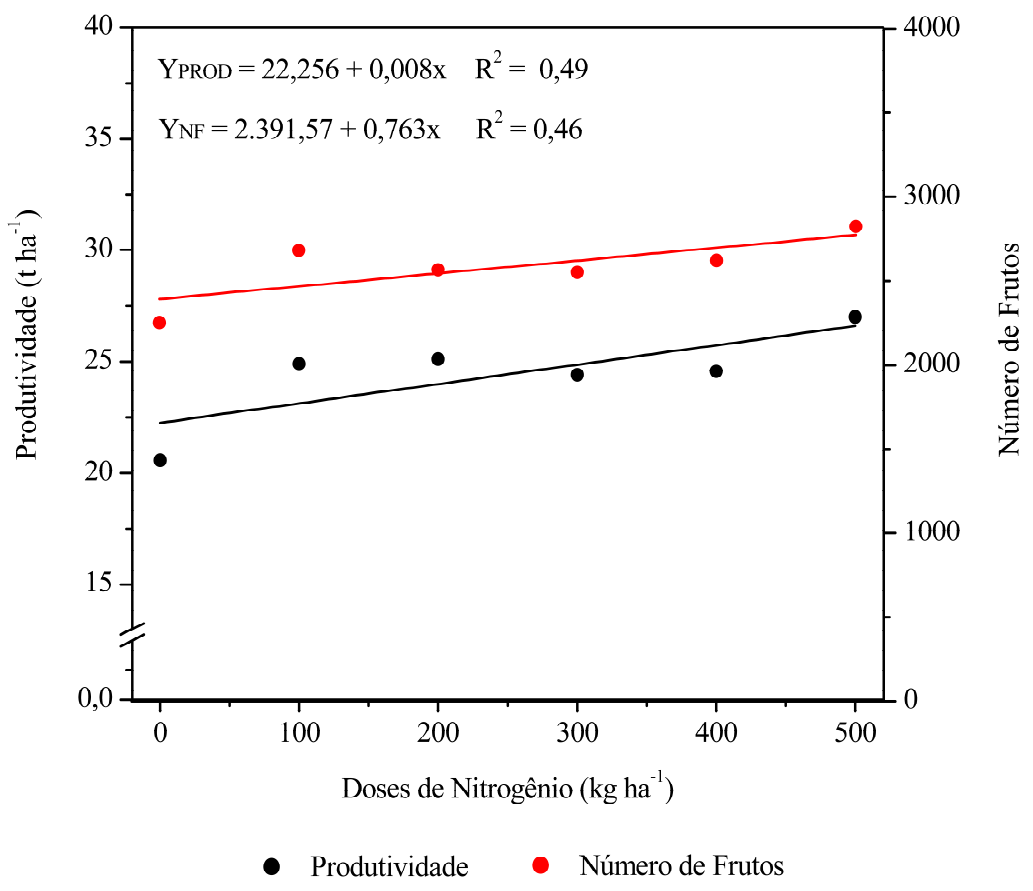

Figura 1. Produtividade $\left(\mathrm{t} \mathrm{ha}^{-1}\right)$ e número de frutos de pimenta malagueta em função de doses de nitrogênio em Alagoas.

Figure 1. Yield $\left(t \mathrm{ha}^{-1}\right)$ and number of chili pepper fruits as a function of the doses of nitrogen in Alagoas.

O nitrogênio participa de forma ativa no metabolismo vegetal e aumenta a eficiência fotossintética das plantas. Na pimenteira, influencia diretamente em características de frutificação da planta e promove altas produtividades (AYODELE et al. 2015). Essa evidência foi corroborada na presente pesquisa, em que a pimenteira respondeu à adubação nitrogenada. Com $100 \mathrm{~kg} \mathrm{ha}^{-1}$ de $\mathrm{N}$, obteve-se ganho produtivo em relação a testemunha de $10,71 \%$, sendo a dose de $500 \mathrm{~kg} \mathrm{ha}^{-1}$ responsável pela maior produtividade verificada, 26,25 tha $\mathrm{t}^{-1}$. AYODELE et al. (2015) verificaram resposta linear da produtividade da pimenteira a doses de N, e sob a dose de $75 \mathrm{~kg} \mathrm{ha}^{-1}$ de $\mathrm{N}$ ocorreu maior retorno produtivo. Enquanto, EKWU et al. (2002) obtiveram uma maior produtividade em cultivo de pimenteira amarela sob uma dose de $150 \mathrm{~kg} \mathrm{ha}^{-1}$ de N. MALAVOLTA et al. (2011) afirmam que a interferência de adubações em cultivos depende não só da cultura, mas também das condições edafoclimáticas, como tipo de solo e disponibilidade hídrica. Logo, respostas produtivas de determinadas culturas, como a pimenteira malagueta, podem ser mais acentuadas a adubação nitrogenada a depender do local de cultivo, manejo e cultivar, fato esse observado nessa pesquisa quando comparado com resultados de outras regiões.

$\mathrm{O}$ número de frutos por planta da pimenta malagueta em função das doses de $\mathrm{N}$ ajustou-se a um modelo linear, a exemplo da variável produtividade (Figura 1). A dose de $500 \mathrm{~kg} \mathrm{ha}^{-1}$ gerou um maior número de frutos por planta (2.773 frutos) ao final do período de colheita. A menor produção de frutos foi verificada no tratamento sem aplicação de $\mathrm{N}$, que resultou numa produção de 2.251 frutos, redução de $18,82 \%$ no número de frutos quando comparado com a maior dose aplicada. AYODELE et al. (2015) afirmam que o $\mathrm{N}$ atua de forma importante na floração e frutificação da pimenteira e interfere no número de frutos por planta. MEDINA-LARA et al. (2008), avaliaram o efeito da adubação nitrogenada em pimenteira na região do México, e verificaram que o aumento da dose de $\mathrm{N}$ gera desenvolvimento precoce da planta, formando um dossel mais uniforme, com maior número de flores e frutos. Tais informações corroboram com os resultados obtidos, em que se verificou que o aumento da dose de nitrogênio gerou aumento linear do número de frutos, o que proporcionou maior produtividade.

O índice SPAD ajustou-se a um modelo quadrático (Figura 2). O maior valor desse índice foi verificado sob a dose de $500 \mathrm{~kg} \mathrm{ha}^{-1}$, sendo 55,28 unidades de índice SPAD. O menor valor foi obtido na testemunha, equivalente a 35,03 unidades de índice SPAD. 




Figura 2. Índice SPAD de pimenta malagueta sob doses de adubação nitrogenada em Alagoas.

Figure 2. SPAD Index of chili peppers fertilized with doses of nitrogen in Alagoas.

O índice SPAD está diretamente relacionado com a intensidade verde das folhas e é comumente utilizado como indicador da concentração de nitrogênio foliar, auxiliando no manejo nutricional de cultivos quanto à adubação nitrogenada (FONTES \& ARAÚJO 2006). Logo, plantas com alto índice SPAD tendem a possuir maior concentração de clorofila, pigmento fotorreceptor da fotossíntese, sendo fotossinteticamente mais eficientes, capazes de produzir maior quantidade de fotoassimilados, consequentemente, sendo mais produtivas. Essas condições foram verificadas nessa pesquisa, plantas sob a dose de $500 \mathrm{~kg} \mathrm{ha}^{-1}$ produziram um maior índice SPAD e obtiveram uma maior produtividade.

\section{CONCLUSÃO}

A dose de $500 \mathrm{~kg} \mathrm{ha}^{-1}$ de nitrogênio proporciona maior índice SPAD, número de frutos e produtividade da pimenta malagueta.

\section{REFERÊNCIAS}

ALLEN RG et al. 1998. Crop evapotranspiration: guidelines for computing crop requirements. Roma: FAO. 328p.

AYODELE OJ et al. 2015. Nitrogen Fertilizer Effects on Growth, Yield and Chemical Composition of Hot Pepper (Rodo). International Journal of Agriculture and Crop Sciences 8: 666-673.

DOORENBOS J \& KASSAM AH. 1986. Efectos del agua sobre el rendimento de los cultivos. Roma: FAO. 212p.

EKWU IG et al. 2002. Effect of plant spacing and nitrogen fertilizer on the growth and yield of pepper (Capsicum annuum

L.). Agro-Science Journal of Tropical Agriculture, Food, Environment and Extension 3: 22-26.

EMBRAPA. 2013. Empresa Brasileira de Pesquisa Agropecuária. Sistema Brasileiro de Classificação de Solos. 3.ed. Brasília: EMBRAPA. 353p.

FONTES PCR \& ARAÚJO C. 2006. Use of chlorophyll meter and plant visual aspect for nitrogen management in tomato fertirrigation. Journal of Applied Horticulture 8: 8-11.

FURLAN JC et al. 2015. Produção de pimenta malagueta em função da adubação nitrogenada e do gel hidroretentor. In: XXXV Congresso Brasileiro de Ciências do Solo. Anais ... Natal: EMPARN.

MALAVOLTA E et al. 2011. Adubos e adubações. São Paulo: Nobel. 200p.

MAHMUD K et al. 2020. Effect of Nitrogen On Growth and Yield of Chili (Capsicum annuum L.) in Roof Top Garden. Turkish Journal of Agriculture-Food Science and Technology 8: 246-251.

MEDINA-LARA $F$ et al. 2008. Influence of nitrogen and potassium fertilization on fruiting and capsaicin content of habanero pepper (Capsicum chinense Jacq). HortScience 43: 1549-1554.

NIMER E. 1977. Geografia do Brasil: Região Nordeste. 2.ed. Rio de Janeiro: IBGE. 421p.

FAO. 2017. Organização das Nações Unidas para Alimentação e Agricultura. Disponível em: http://www.fao.org/faostat/en/\#data/QC. Acesso em: 11 nov. 2019.

PINTO CMF et al. 1999. A cultura da pimenta (Capsicum sp.). Belo Horizonte: EPAMIG. 39p. (Boletim Técnico 56).

TAIZ L et al. 2017. Fisiologia e desenvolvimento vegetal. 6.ed. Porto Alegre: Artmed. 858p. 